\title{
SYNTHETIC ANALYSIS OF MODEL SMOOTHING'S INFLUENCE ON RAY PATH, TRAVELTIME AND MIGRATION IMAGING
}

\author{
SUN, H. - SUN, J. G. ${ }^{*}$ - HAN, F. X. - SUN, Z. Q. - LIU, Z. Q. ${ }^{*}-$ LIU, M. C. ${ }^{*}-$ HUANG, X. G. \\ College for Geoexploration Science and Technology, Jilin University \\ No.938, Ximinzhu Street, Changchun, Jilin Province, 130026, P. R. China \\ (phone: +04-31-8592-3862; fax: +04-31-8592-3862) \\ *Corresponding authors \\ e-mail: sun_jg@jlu.edu.cn; liuzq13@mails.jlu.edu.cn; liu_mch@163.com \\ (Received $22^{\text {nd }}$ Jul 2016; accepted $27^{\text {th }}$ Feb 2017)
}

\begin{abstract}
Smoothing process might change the value of grid nodes in the migration model, which is essential to seismic imaging. We will study the influence of model smoothing on ray path and traveltime as well as their connections with the migration imaging. A deep-water velocity model is employed for the calculation. The numerical analysis shows that model smoothing has a significant influence on ray path and traveltime, these differences can affect the migration images. Optimal smoothing process is beneficial to achieve high-quality migration images, while inappropriate model smoothing could even change the shape of real geology body and lead to wrong geology structures in the migration sections.
\end{abstract}

Keywords: velocity smoothing, seismic imaging, traveltime, ray path, wave propagation

\section{Introduction}

Many ray-based migration methods are widely employed in seismic exploration (Hill, 1990; Keho and Beydoun, 1998; Palo et al., 2016). However, these methods are put forward based on high-frequency approximation theory which requires the value of velocity ranging steadily in a certain wavelength (Geoltrain and Brac, 1993). Often migration model cannot meet this requirement, so it need to be smoothed (Červený, 2005).

Smoothing, on the other hand, will change original velocity model. While pre-stack depth migration is sensitive to velocity, even a tiny velocity error can decreasee the imaging quality (Versteeg, 1993; Bevc, 1997; Zhu et al., 1998; Herron, 2000). So the processing of smoothing need to be carefully treated. Gray (2000) contrasted three complex models' migration results to learn the model smoothing's influence on migration imaging. Alde et al. (2002) determined the optimally smoothed slowness model for ray-tracing based migration methods. He uesed multiple valued traveltime tables to reach his goal. Pacheco and Larner (2005) futher studied the model smoothing's effect on seimic migration imaging. They maintain the view that the optimal amount of smoothing is difficult to be determined. Han et al. (2008) employed different smoothing operator in his research and demostrated that convolution smoothing operator is more effective for smoothing processing.

In the following sections, a deep-water velocity model is employed for the numerical experiments. We first introduce the theoretical basis of smoothing for velocity model and then analyze the influences of model smoothing on ray path and traveltime. The migration results are also provided to demonstrate model smoothing's effect on seimic imaging. Meanwhile the connections between ray path, traveltime and migration imaging are discussed. 


\section{Methods}

\section{Theoretical basis of smoothing for velocity model}

In this part, the theoretical basis of model smoothing will be analyzed from the aspects of solving ray-tracing equations and high-frequency approximation theory.

Necessity of model smoothing for solving ray tracing equations

Different ray-based methods can obtain ray information in different ways. Though these methods' implementations are different but they all have the same requirement for solving ray tracing equations. Ray tracing equations can be further divided into two types, namely kinematic ray tracing (KTR) and dynamic ray tracing (DTR) (Červený, 2005; Coman and Gajewski, 2005):

$$
\begin{gathered}
\left\{\begin{array}{l}
\frac{d x_{i}}{d \tau}=v^{2} p_{i}, i=1,2,3 \\
\frac{d p_{i}}{d \tau}=-\frac{1}{v} \frac{\partial v}{\partial x_{i}}, i=1,2,3
\end{array}\right. \\
\left\{\begin{array}{l}
\frac{d \boldsymbol{Q}}{d \tau}=v^{2} \boldsymbol{P} \\
\frac{d \boldsymbol{P}}{d \tau}=-\frac{1}{v} \boldsymbol{V} .
\end{array}\right.
\end{gathered}
$$

$x_{i}$ describes position components in Cartesian coordinate system; $v$ expresses velocity; $p_{i}$ indicates slowness component $. \boldsymbol{Q}, \boldsymbol{P}, \boldsymbol{V}$ can be further described as follows:

$$
\boldsymbol{Q}=\left[\begin{array}{ll}
Q_{11} & Q_{12} \\
Q_{21} & Q_{22}
\end{array}\right], \boldsymbol{P}=\left[\begin{array}{ll}
P_{11} & P_{12} \\
P_{21} & P_{22}
\end{array}\right], \boldsymbol{V}=\left[\begin{array}{ll}
V_{11} & V_{12} \\
V_{21} & V_{22}
\end{array}\right]
$$

Each component in the matrix can be expressed in following formulas:

$$
Q_{I J}=\frac{\partial q_{I}}{\partial \gamma_{J}}, P_{I J}=\frac{\partial p_{I}}{\partial \gamma_{J}}, V_{I J}=\frac{\partial v}{\partial q_{I} \partial q_{J}}
$$

$q_{I}(I=1,2)$ is central ray coordinate while $\gamma_{J}(J=1,2)$ is ray parameters alongside each ray.

It can be known from the above equations that velocity model is required to have the second-order continual derivatives. However, the real velocity models often cannot meet the requirement. It is necessary to smooth these models to guarantee the equations' solving conditions.

\section{Necessity of model smoothing for high-frequency approximation}

High-frequency approximation is the precondition of ray theory (Červený et al., 1977). Therefore, relative velocity variation $\Delta v / v$ in a certain wavelength range cannot 
be too large in inhomogeneous medium. Supposing $f=50 \mathrm{~Hz}$ is dominant frequency in seismic exploration and the average velocity is $3000 \mathrm{~m} / \mathrm{s}$. Then the wave length is about $60 \mathrm{~m}$. If $\Delta v / v$ is on the order of magnitude of $10^{-2}$, then velocity gradient is demanded within $0.5 \mathrm{~m} / \mathrm{s}$. However, lots of migration model cannot meet this requirement. So the velocity models should be smoothed to decrease the relative velocity variation.

\section{Selecting smooth operators}

Model smoothing is realized by changing velocity models with smooth operators for many times. Han et al. (2008) discussed three normal operators (namely, hree-point smoothing operator, five-point smoothing operator and convolution smoothing operator) to assess their influence on seismic traveltime and ray path. The conclusion is that convolution smoothing operator is better for model smoothing. The convolution smoothing operator is also chosen in this paper as is shown below:

$$
v_{i, j}^{k+1}=\frac{1-\omega}{2} v_{i-1, j}^{k}+\omega v_{i, j}^{k}+\frac{1-\omega}{2} v_{i+1, j}^{k}
$$

$v_{i, j}^{k}$ is the velocity of grid node $\left(x_{i}, z_{j}\right)$ after $k$ times smoothing; $\omega$ varies from 0 to 1 , which is choosing as 0.5 in the paper.

\section{Results}

\section{Construction of velocity model in deep water}

The paper analyzes differences between ray path, traveltime and migration quality based on the velocity model in deep water which is demonstrated in Fig. 1. The size of the model is $1501 \times 1601$; The grid spacing of the model is $4 \mathrm{~m} \times 4 \mathrm{~m}$; The seawater depth in the model varies from $2800 \mathrm{~m}$ to $3500 \mathrm{~m}$. Seawater velocity has a constant value of $1500 \mathrm{~m} / \mathrm{s}$. The velocity value in layers below seawater are respectively $2500 \mathrm{~m} / \mathrm{s}$, $2700 \mathrm{~m} / \mathrm{s}, 2900 \mathrm{~m} / \mathrm{s}, 3000 \mathrm{~m} / \mathrm{s}, 3100 \mathrm{~m} / \mathrm{s}, 3300 \mathrm{~m} / \mathrm{s}$, and $3500 \mathrm{~m} / \mathrm{s}$ from up to down. The velocity value of embedded body is $3300 \mathrm{~m} / \mathrm{s}$.

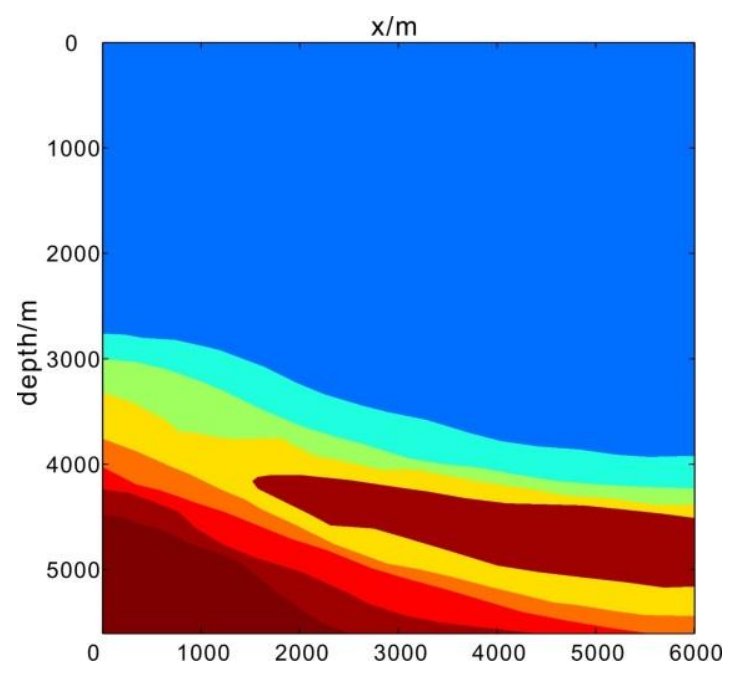

Figure 1. Velocity model in deep water 


\section{Effect of smoothing on ray path in deep water velocity model}

Different smoothing amount produces differences in original model. This part will show effects of model smoothing on ray path in deep water velocity model.

Fig. 2a-2f demonstrate corresponding ray path at the same shot point when smoothing amount is respectively $5,10,20,30,50$ and 100 . The number of rays starting from the source is 31 . The angle between adjacent rays is $2^{\circ}$ and emergence angles range from $-60^{\circ}$ to $0^{\circ}$. It can be seen from the figures that the smoothing amount can not influence ray path before ray enters layers because of the same seawater velocity which is not influenced by smoothing. However, the condition is quite different after the rays enter the layers. When smoothing amount is 5, 12 rays travel through the high speed body. It increases up to 15 rays when smoothing amount is 10 . Furthermore, rays at the emergence angle of $8^{\circ}$ and $10^{\circ}$ stop intersecting. While smoothing number is 20 , paths of rays at emergence angle of $-28^{\circ},-26^{\circ}$ and $-24^{\circ}$ change seriously in layers and rays at the emergence angle of $-4^{\circ}$ and $-6^{\circ}$ intersect under the high speed body. Rays at the emergence angle of $-28^{\circ} 、-26^{\circ}$ and $-24^{\circ}$ experience another big change in path when smoothing amount is 30 . When the smoothing amount is 50,16 rays travel through the high speed body. Meanwhile, number of ray turning back to seawater decreases. Finally, when the smoothing amount is 100,17 rays travel through the high velocity body without any intersection.

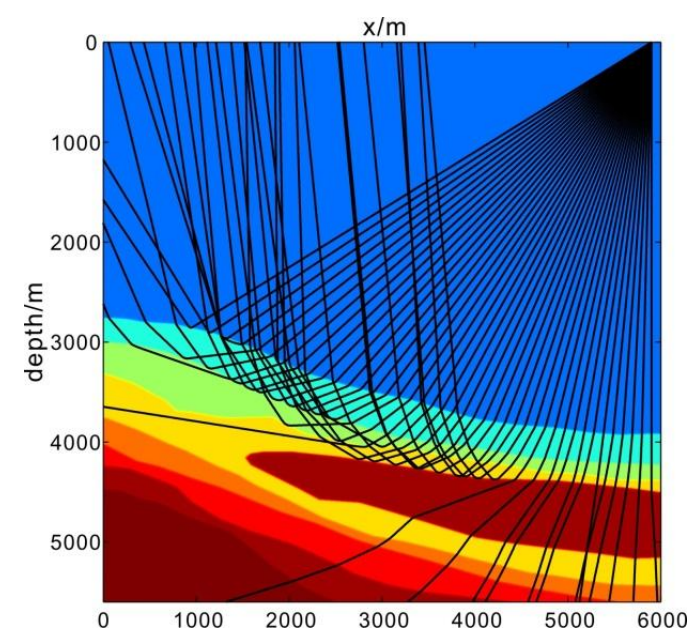

(a)

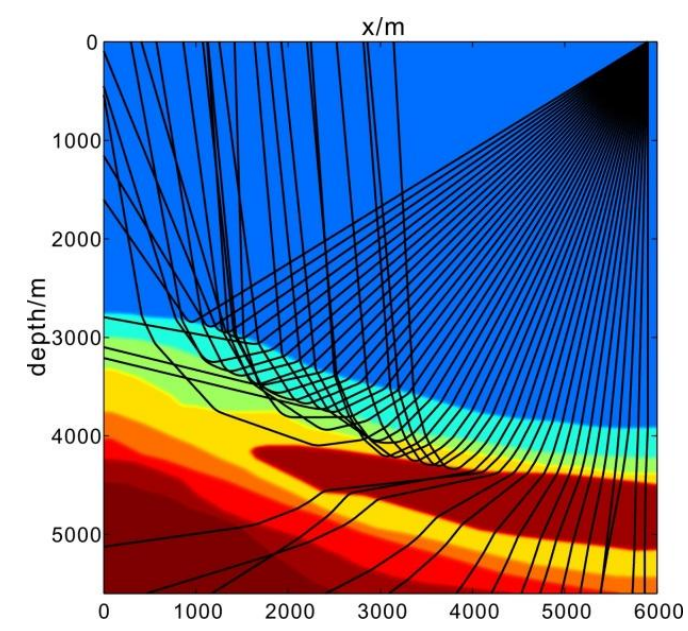

(c)

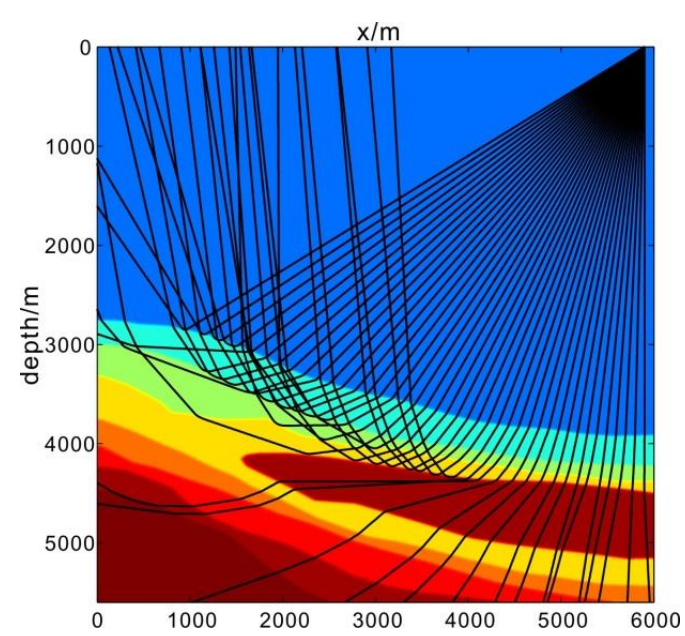

(b)

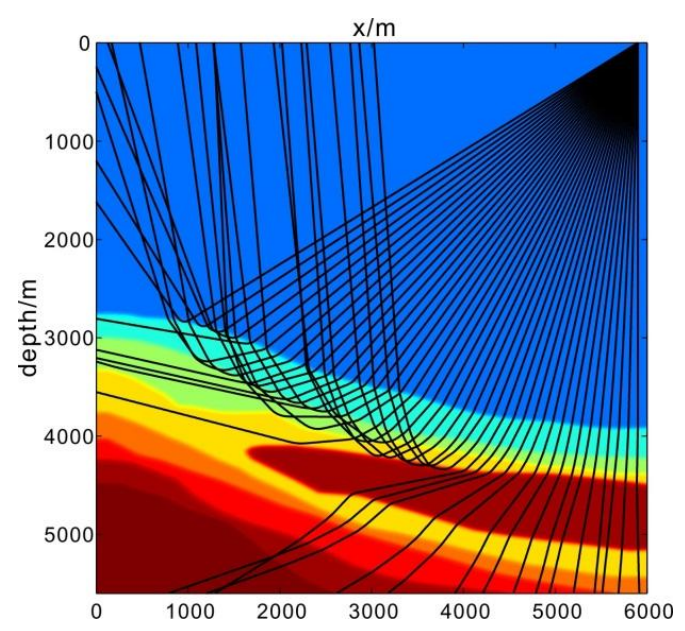

(d) 


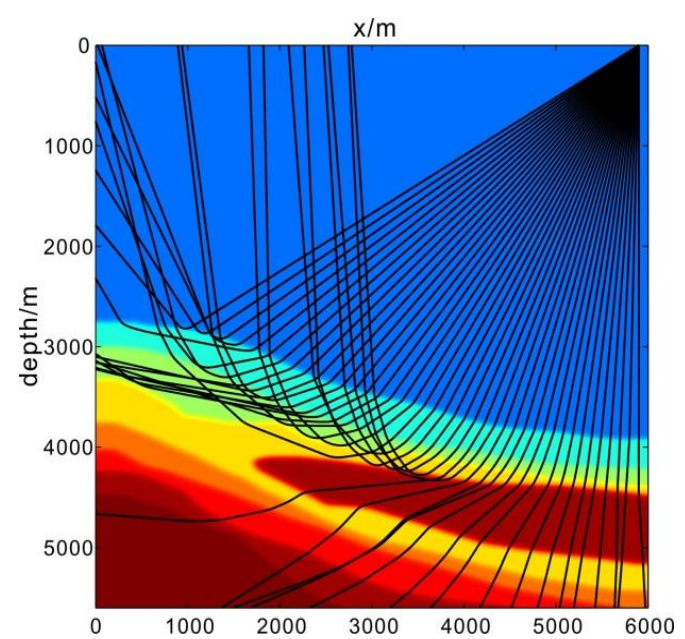

(e)

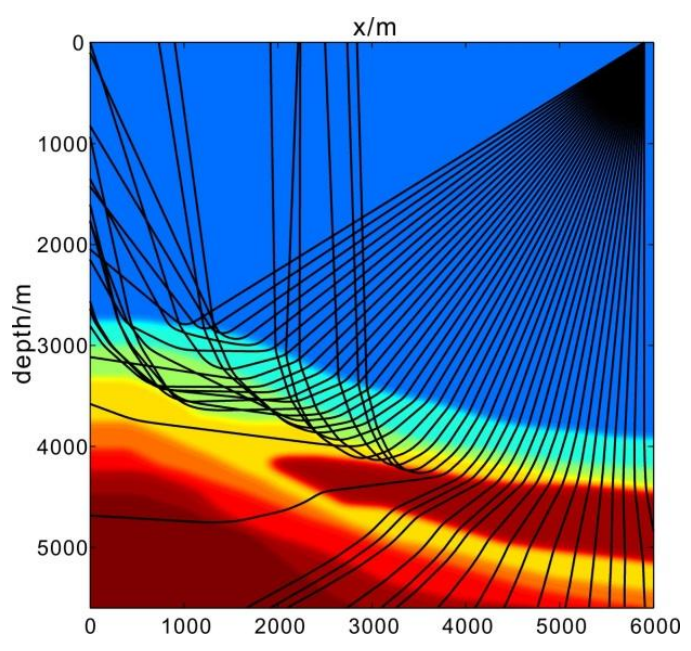

(f)

Figure 2. Ray paths under different amount of smoothing, (a)ray paths when the amount is 5, (b)ray paths when the amount is 10, (c)ray paths when the amount is 20, (d)ray paths when the amount is 30, (e)ray paths when the amount is 50, (f)ray paths when the amount is 100

\section{Effect of smoothing on traveltime in deep water velocity model}

For the reason that some grid nodes' velocity value are different, the traveltime can also be influenced. It will be studied in detail in this part. Fig. $3 a$ illustrates isochron distribution of original model without smoothing. Figure $3 b-3 g$ respectively depicts relative error of traveltime with the result obtained in Fig. $3 a$ when smoothing number is 5, 10, 20, 30, 50 and 100. It has to be clarified that the relative error is just a parameter to describe difference of traveltime without any meaning of assessment. Generally, traveltime error increases with smoothing amount in layers of deep water model. The maximum of traveltime error is respectively $1.71 \%$, $3.18 \%$, $6.14 \%$ and 9.04 when smoothing number is $5,10,20$ and 30. Then maximum of traveltime error breakthroughs $1 \%$ as the smoothing amount increasing to 50 . When smoothing amount reaches 100 , the maximum is even up to $2.5 \%$. What's more, we can find that high relative error region which is in red color occupies a large proportion in these figures. Fig. 4 shows traveltime curves with different smoothing amount at the depth of $5500 \mathrm{~m}$. It can be concluded that the larger the smoothing amount is, the higher the corresponding traveltime error is. Difference of traveltime at the same position can reach as high as $79 \mathrm{~m} / \mathrm{s}$ or at least $33 \mathrm{~m} / \mathrm{s}$ when smoothing amount is 100 .

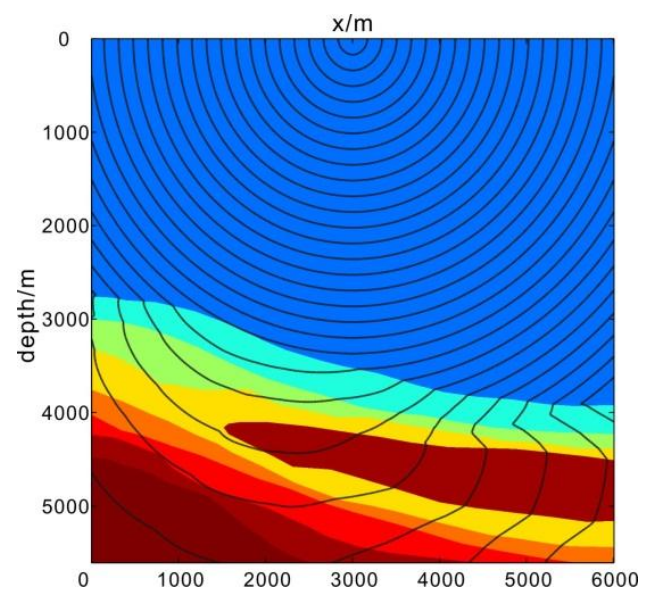

(a)

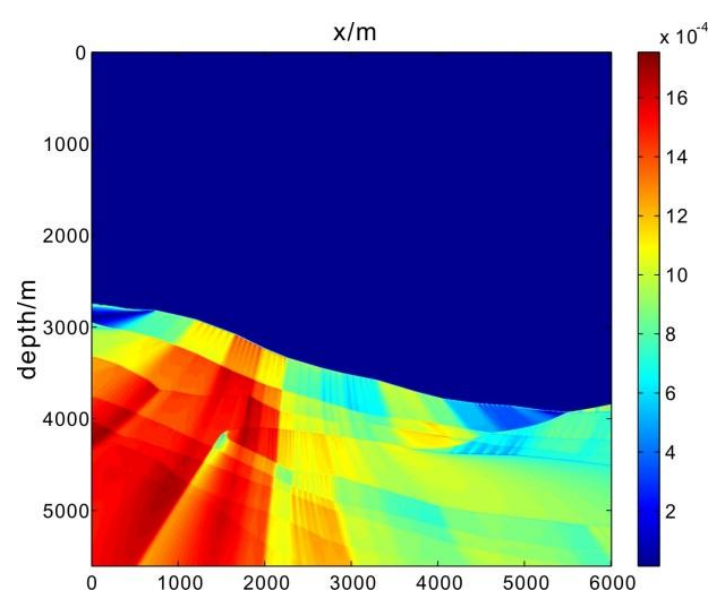

(b) 


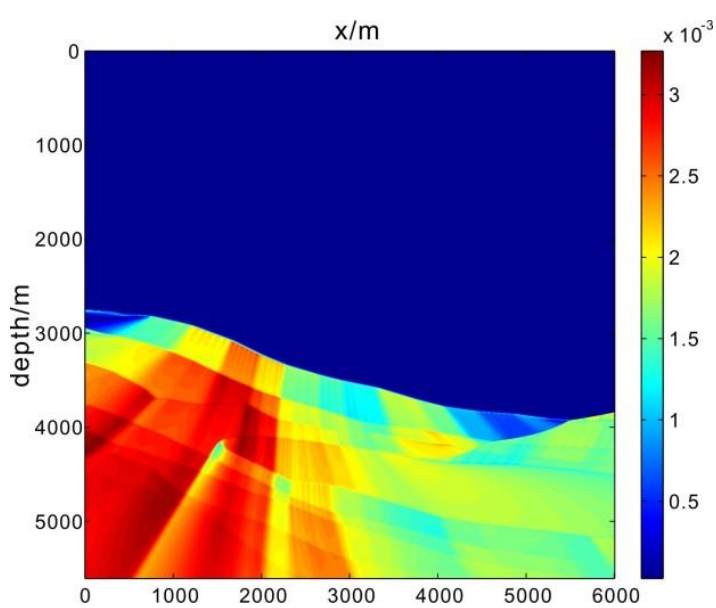

(c)

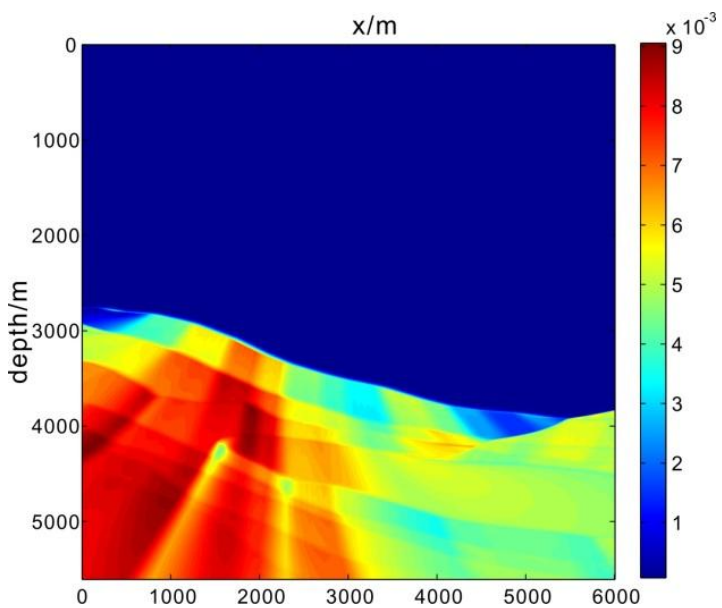

(e)

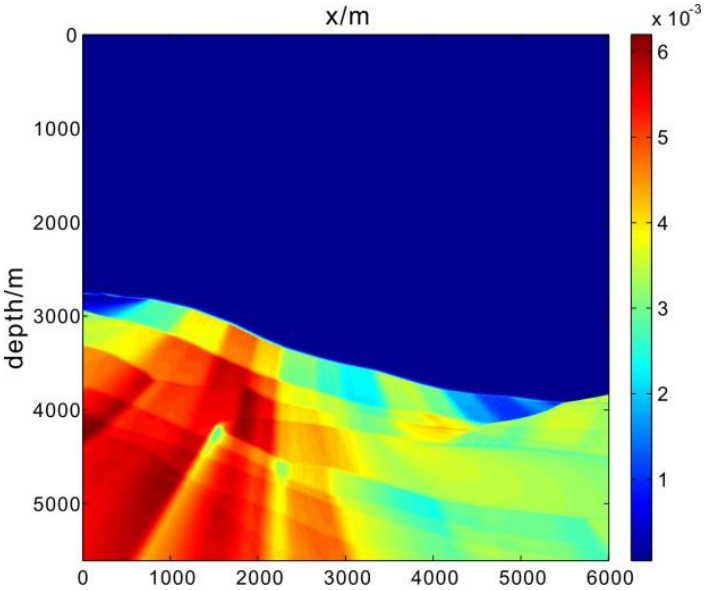

(d)

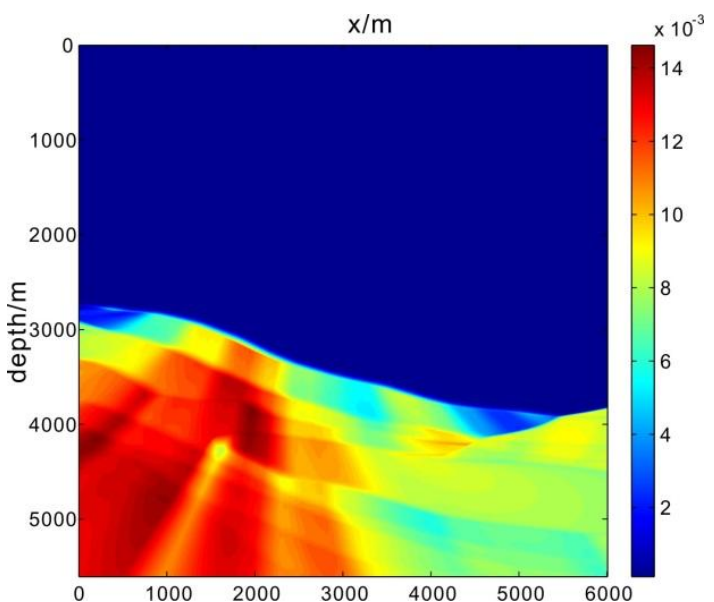

(f)

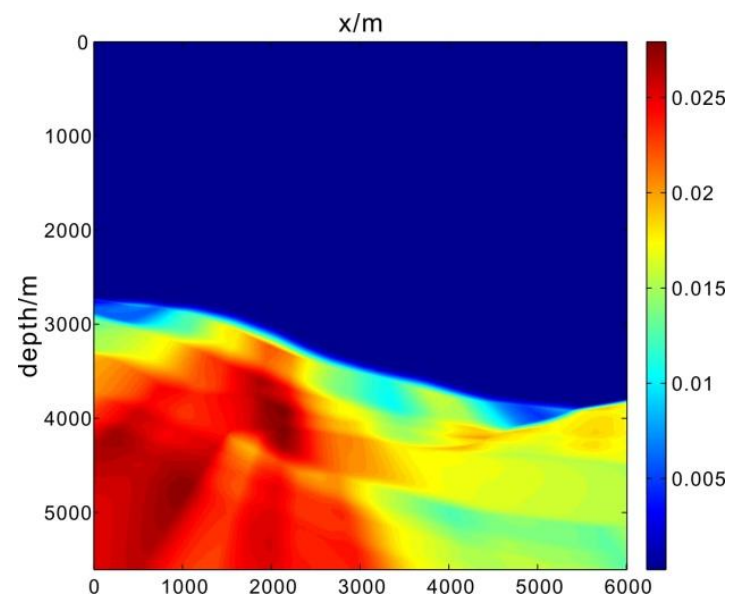

(g)

Figure 3. Isochron distribution of original model and relative error of traveltime under different amount of smoothing, (a) isochron distribution of original model, (b) relative error of traveltime when the amount is $5,(c)$ relative error of traveltime when the amount is $10,(d)$ relative error of traveltime when the amount is 20, (e) relative error of traveltime when the amount is 30, (f)relative error of traveltime when the amount is 50, $(\mathrm{g})$ relative error of traveltime when the amount is 100 


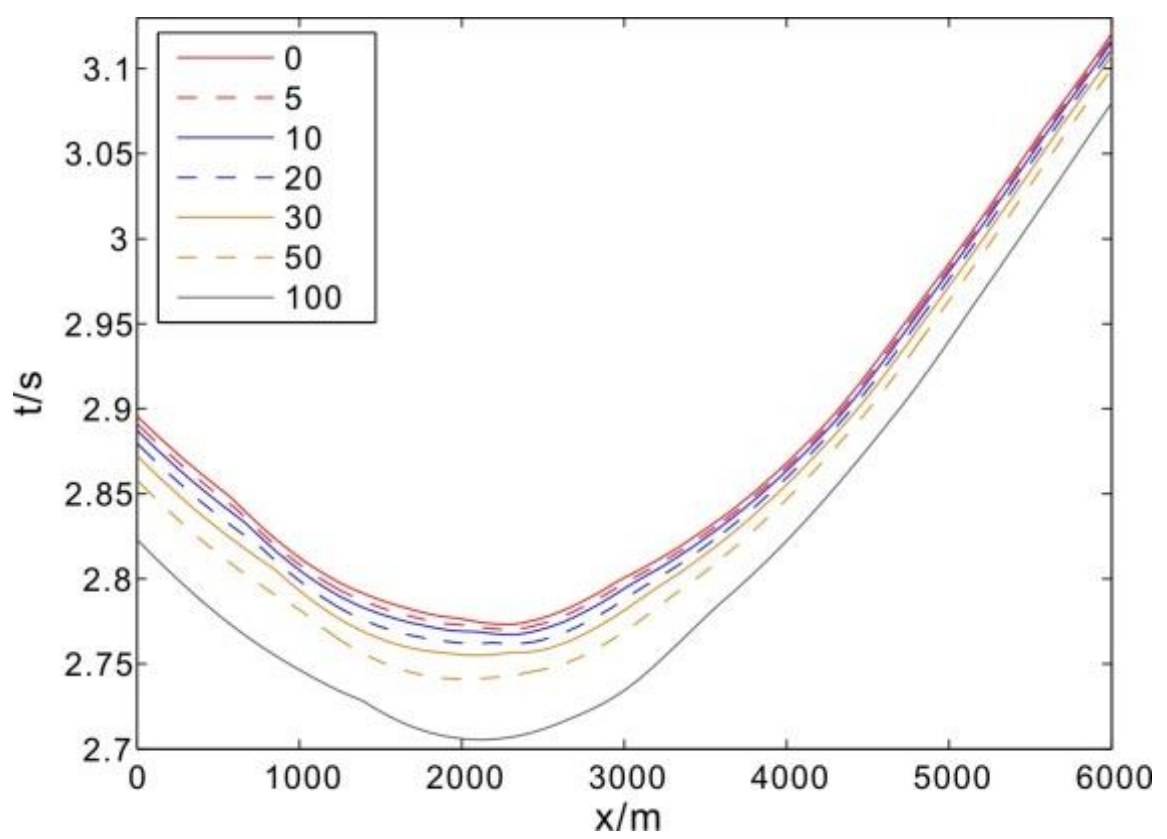

Figure 4. Corresponding traveltime curve with different smoothing amount at the depth of $5500 \mathrm{~m}$

\section{Effect of model smoothing on migration quality in deep-water velocity model}

The previous sections discussed model smoothing's influence on ray path and traveltime. In this part, effects of smoothing processing on migration quality of deepwater velocity model is analyzed. Kirchhoff beam prestack depth migration is choosing as the migration method. The original model is simulated to obtain the seismic records first. Fig. $5 a$ demonstrates migration of original velocity model and Fig. $5 b-5 g$ respectively show the results of migration model which has experienced 5 , $10,20,30,50$ and 100 times smoothing processing. It can be summarized from the figures that no matter how many the smoothing number is, the interface between seawater and strata can be recognized in the migration section. It is in consistency with the analysis on ray path and seismic traveltime. In the migration section without smoothing processing, only the first interface can be recognized. It achieves quite a good result when smoothing processing is processed for 5 or 10 times. The true geology structure and interfaces can be shown clearly in the migration sections. When the smoothing amount adding up to 20 , the migration quality, however, decreases obviously. Migration energy can not be well focused on interfaces and the middle of the third interface can not be recognized. As smoothing amount increases to 30, decrease of migration quality continues. Furthermore, the location of interfaces starts moving upwards. The deeper the interface location is, the higher it moves and the thickness of high velocity body is quite little. While smoothing has been processed for 50 times, interfaces in the figure has been distorted severely. Therefore, the geology structure obtained from the migration section differentiates a lot from the origin one. When the smoothing amount reaches 100, all the interface lines cannot be distinguished except for the line between seawater and strata. It can be concluded that moderate amount of smoothing can elevate the quality of migration imaging. 


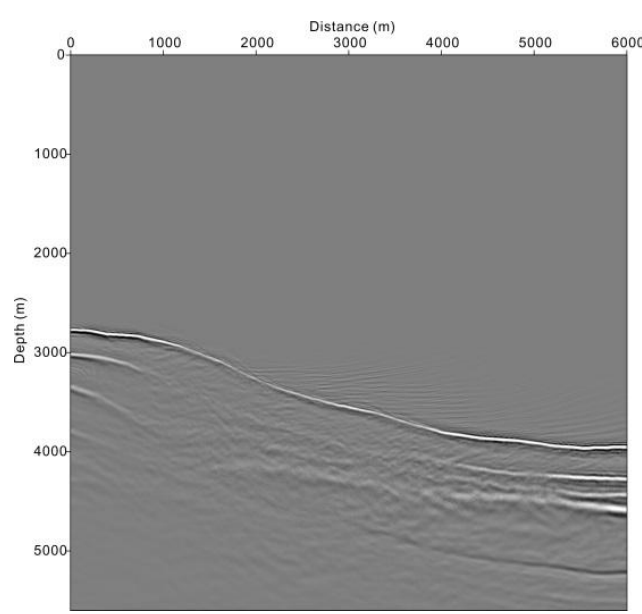

(a)

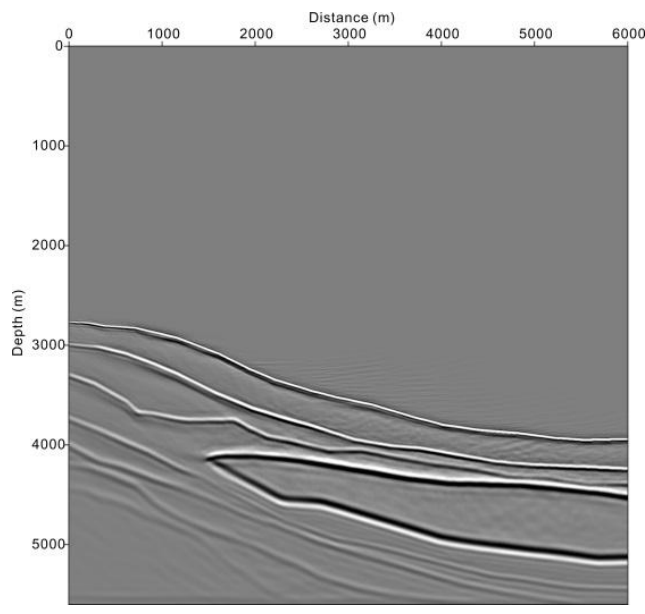

(c)

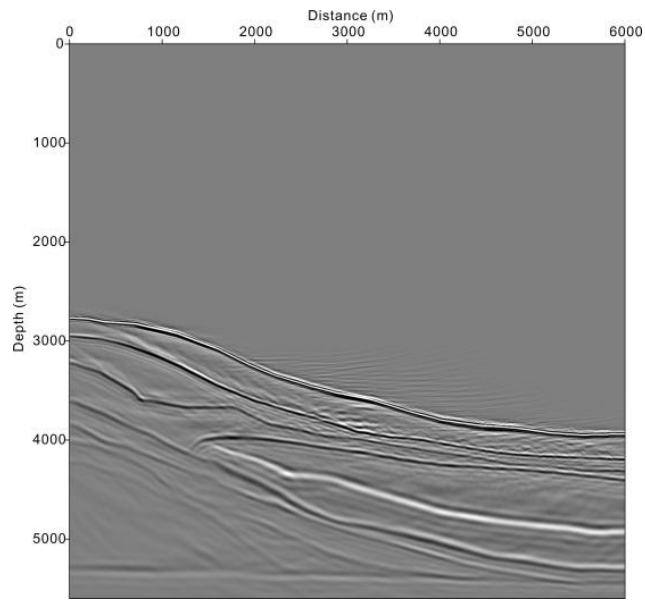

(e)

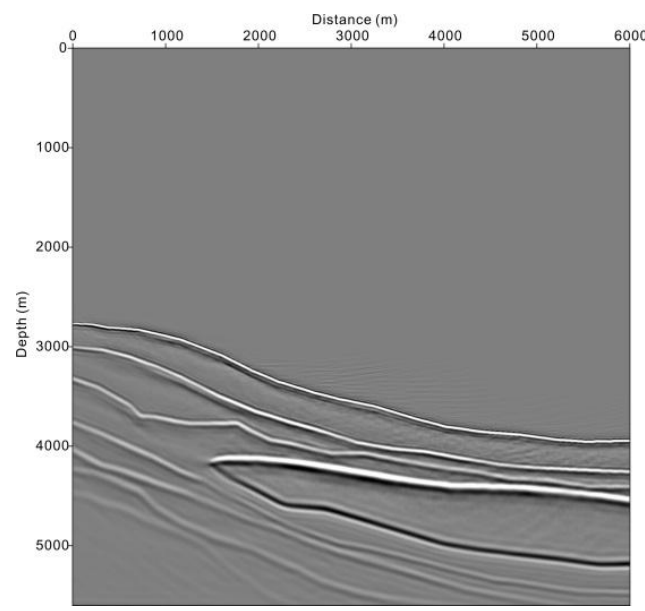

(b)

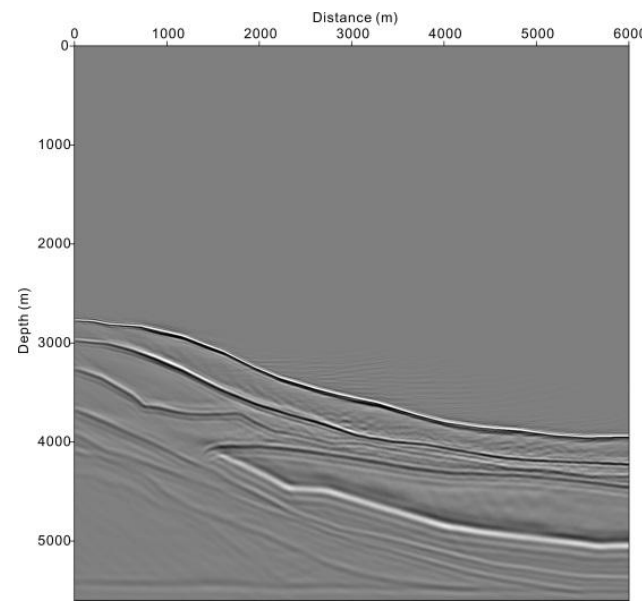

(d)

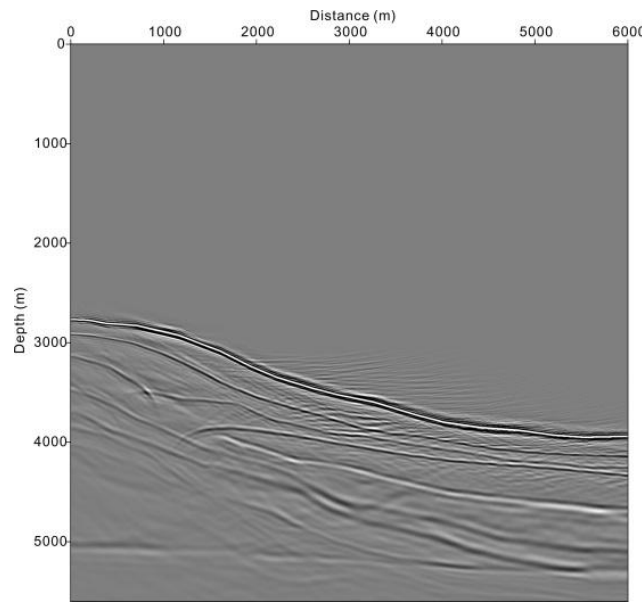

(f) 


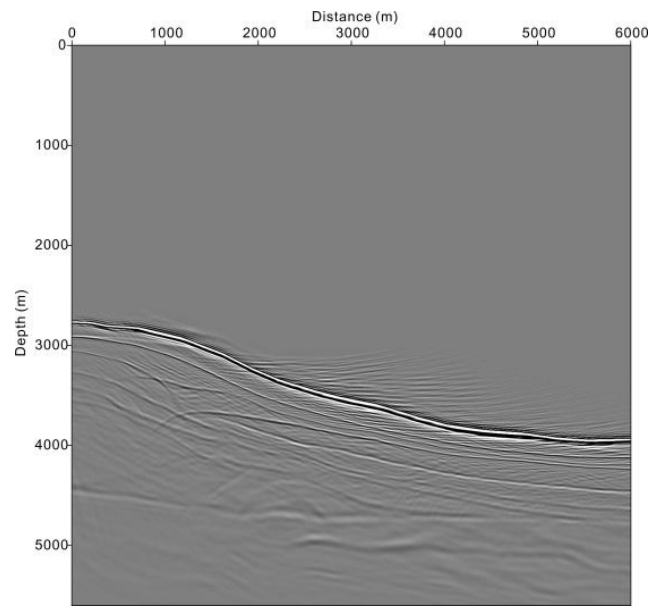

(g)

Figure 5. Migration results under different amount of smoothing, (a) migration result when the amount is 0 , (b) migration results when the amount is 5, (c) migration result when the amount is 10 , (d) migration result when the amount is 20 , (e) migration result when the amount is 30 , $(f)$ migration result when the amount is 50, $(\mathrm{g})$ migration result when the amount is 100

\section{Discussion}

We have smoothed the velocity model to obtain different migration results with different smoothing times. Different smoothed migration model can result in different ray tracing results which contains the information of ray path and traveltime. The numerical analysis of deep-water model shows that smoothing process can affect the quality of stacked images, optimal smoothing times can generate a better imaing result than one with too many or too few smoothing times. Therefore, we should pay more attention to model smoothing in practice.

Acknowledgements. This study was supported financially by the National Natural Science Foundation of China (41274120, 41404085, 41504084).

\section{REFERENCES}

[1] Alde, D. M., Fehler, M. C., Hildebrand, S. T. (2002): Determining the optimally smoothed slowness model for ray-tracing based migration using multiple-valued traveltime tables. -72th Technical Program Meeting, SEG, Society of Exploration Geophysicists: 1168-1171.

[2] Bevc, D. (1997): Imaging complex structures with semirecursive Kirchhoff migration. Geophysics 62(2): 577-588.

[3] Červený, V. (2005): Seismic ray theory. - Cambridge university press, England.

[4] Červený, V., Molotkov, I. A., Molotkov, I. A. (1977): Ray method in seismology. Univerzita Karlova, Chech.

[5] Coman, R., Gajewski, D. (2005): Traveltime computation by wavefront-orientated ray tracing. - Geophysical prospecting 53(1): 23-36.

[6] Geoltrain, S., Brac, J., (1993): Can we image complex structures with first-arrival traveltime?. - Geophysics 58(4): 564-575.

[7] Gray, S. H. (2000): Velocity smoothing for depth migration: How much is too much?. 70th Annual International Meeting, SEG, Expanded Abstracts: 1055-1058. 
[8] Han, F. X., Sun, J. G., Sun, Z. Q. (2008): Influence of smooth operator on travel-times and ray paths of seismic waves. - Oil Geophysical Prospecting 43(4): 405-409.

[9] Herron, D. A. (2000): Pitfalls in seismic interpretation: Depth migration artifacts. - The Leading Edge 19(9): 1016-1017.

[10] Hill, N. R. (1990): Gaussian beam migration. - Geophysics 55(11): 1416-1428.

[11] Keho, T. H., Beydoun, W. B. (1988): Paraxial ray Kirchhoff migration. - Geophysics 53(12): 1540-1546.

[12] Pacheco, C., Larner, K. (2005): Velocity smoothing before depth migration: Does it help or hurt? - Houston 2005 Annual Meeting, SEG, Expanded Abstracts: 1970-1973.

[13] Palo, M., Tilmann, F., Schurr, B. (2016). Applicability and Bias of VP/VS Estimates by P and S Differential Arrival Times of Spatially Clustered Earthquakes. - Bulletin of the Seismological Society of America 106(3): 1055-1063.

[14] Versteeg, R. J. (1993): Sensitivity of prestack depth migration to the velocity model. Geophysics 58(6): 873-882.

[15] Zhu, J., Lines, L., Gray, S. (1998): Smiles and frowns in migration/velocity analysis. Geophysics 63(4): 1200-1209. 